\title{
Membrane based scandium extraction from industrial waste
}

\author{
S. Hedwig ${ }^{1}$, E. C. Constable ${ }^{2}$, M. LenZ ${ }^{1}$
}

${ }^{1}$ FHNW, Institute for Ecopreneurship, Switzerland, Hofackerstrasse 30, CH-4132 Muttenz

${ }^{2}$ University of Basel, Department of Chemistry, Switzerland, Mattenstrasse 24a, CH-4058

Aviation contributes to climate change through $\mathrm{CO}_{2}$ emissions and technological solutions are required to reduce its environmental impact. A big step forward could be the enhanced use of scandium (Sc), as it allows the construction of up to $20 \%$ lighter aircraft. Although this has been technically possible for decades, the application of $\mathrm{Sc}$ is limited by an unreliable and insufficient supply chain. To overcome this barrier, new approaches for the production of Sc are sought. ${ }^{[1]}$ In this study, polymer inclusion membranes (PIMs) were crafted and optimized to enable selective Sc separation from $\mathrm{TiO}_{2}$ production waste. The optimal membrane composition in terms of Sc extraction quantity and selectivity was determined experimentally. The best membrane was used in diffusion experiments between a Sc-containing feed solution and different receiving phases (mineral acids capable of taking up Sc). Scandium was absorbed quantitatively ( $>95 \%)$, transported and released ( $>40 \%)$ into the receiving solution. The mole fraction of Sc among the dissolved metals increased two orders of magnitude from the feed to the receiving phase, showing an outstanding selectivity. This method was successfully applied on various acidic multi-element model solutions, but also on real acid waste from white pigment production. These results suggest PIMs as a convenient solution to exploit a previously untapped source of Sc, which may help to boost Sc based technologies.

\section{Acknowledgements}

This project has received funding from the EC H2020

Programme under grant agreement $n^{\circ} 730105$ (SCALE).

\section{References}

[1] Remmen, K., et al., Environ. Sci. Water Res. Technol., 05, 1683-1688. 